\title{
DIOPHANTINE EQUATIONS WITH PRODUCTS OF CONSECUTIVE MEMBERS OF BINARY RECURRENCES
}

\author{
ATTILA BÉRCZES, YURI F. BILU, AND FLORIAN LUCA
}

\begin{abstract}
We prove a finiteness result for the number of solutions of a Diophantine equation of the form $u_{n} u_{n+1} \cdots u_{n+k} \pm 1= \pm u_{m}^{2}$, where $\left\{u_{n}\right\}_{n \geq 1}$ is a binary recurrent sequence whose characteristic equation has roots which are real quadratic units.
\end{abstract}

\section{INTRODUCTION}

Let $\left\{F_{n}\right\}_{n \geq 1}$ be the Fibonacci sequence given by $F_{1}=F_{2}=1$ and $F_{n+2}=$ $F_{n+1}+F_{n}$ for all $n \geq 1$. In [6], inspired by the still unsolved problem of Brocard and Ramanujan asking to determine all positive integer solutions $(n, x)$ of the Diophantine equation

$$
n !+1=x^{2}
$$

Marques fixed a positive integer $t$ and showed that the Diophantine equation

$$
F_{1} \cdot F_{2} \cdots F_{n}+1=F_{m}^{t}
$$

has only finitely many positive integer solutions $(n, m)$. He also computed all solutions when $t \in[1,10]$. The fact that the above equation has only finitely many solutions with $t$ a variable as well follows as a byproduct of the results

\footnotetext{
A.B. was supported by the University of Debrecen, by a János Bolyai Scholarship of the Hungarian Academy of Sciences, and by grants K100339 and NK104208 of the Hungarian National Foundation for Scientific Research. F. L. worked on this paper when he visited the University of Debrecen in Juy 2016 and while he visited the Max Planck Institute for Mathematics in Bonn in 2017. He thanks both these institutions for hospitality and support. He was also supported by grants CPRR160325161141 and an A-rated researcher award both from the NRF of South Africa and by grant no. 17-02804S of the Czech Granting Agency.

2010 Mathematics Subject Classification: 11B39, 11D61.

Keywords and Phrases: Diophantine equations, binary recurrences, applications of linear
} forms in logarithms. 
from [2], where it was shown among other things that the largest integer solution $(k, \ell, m, n)$ of

$$
\left|F_{n} F_{n+1} \cdots F_{n+k-1}-F_{m}^{\ell}\right| \leq 100 \quad \text { with } \quad k \geq 1, \ell \geq 1, m \geq 3, n \geq 3
$$

(and $m \neq n$ when $k=\ell=1$ ) is

$$
\left|F_{9} \cdots F_{13}-F_{11}^{5}\right|=89 .
$$

Here by largest solution it is understood the solution with maximal value of $\max \left\{F_{n} F_{n+1} \cdots F_{n+k-1}, F_{m}^{\ell}\right\}$. This maximal value is 5584059449. Szalay [9], solved completely the Diophantine equation

$$
G_{n_{1}} G_{n_{2}} \cdots G_{n_{k}}+1=G_{m}^{2}
$$

in positive integer unknowns $k, m$ and $n_{1}<n_{2}<\cdots<n_{k}$ where $\left\{G_{n}\right\}_{n \geq 1}$ is in a certain family of Lucas sequences including the Fibonacci sequence. Further generalizations of this problem were considered by Szikszai [10].

The proofs from [6], [9] and [10] all have in common the fact that they use the existence of primitive divisors for terms of Lucas sequences. Here we study the same question (or a variant of) but for a very different kind of sequence $\left\{u_{n}\right\}_{n \geq 1}$ which we now describe. We assume that $\left\{u_{n}\right\}_{n \geq 1}$ is a binary recurrent sequence of integers satisfying $u_{n+2}=r u_{n+1}+s u_{n}$, for all $n \geq 1$, where $r$ and $s$ are fixed nonzero integers. We assume that the characteristic equation $x^{2}-r x-s=0$ has two roots $\alpha \neq \beta$ (so $\Delta=r^{2}+4 s \neq 0$ ) and that $\alpha / \beta$ is not a root of 1 . Such sequences are called nondegenerate. In this case it is known that

$$
u_{n}=c \alpha^{n}+d \beta^{n} \quad \text { holds for all } \quad n \geq 1,
$$

where $c$ and $d$ can be computed in terms of $u_{1}, u_{2}$. In fact,

$$
(c, d)=\left(\frac{u_{2}-\beta u_{1}}{\alpha^{2}-\alpha \beta}, \frac{u_{2}-\alpha u_{1}}{\beta^{2}-\alpha \beta}\right) .
$$

We further assume that $s= \pm 1$. The above restrictions insure that $\Delta>0$ so $\alpha$ and $\beta$ are real. We label these roots in such a way that $|\alpha|>1>|\beta|$ (note that $\left.\beta=-s \alpha^{-1}= \pm \alpha^{-1}\right)$. We find it convenient to use the recurrence for $\left\{u_{n}\right\}_{n \geq 1}$ and define $u_{0}$ such that $u_{2}=: r u_{1}+s u_{0}$. That is, $u_{0}:=s^{-1}\left(u_{2}-r u_{1}\right)$, which is an integer since $s \in\{ \pm 1\}$. Then formula (1.1) holds with $n=0$ as well, while (1.2) can be replaced by the somewhat simpler looking expression

$$
(c, d)=\left(\frac{u_{1}-\beta u_{0}}{\alpha-\beta}, \frac{u_{1}-\alpha u_{0}}{\alpha-\beta}\right) .
$$


Classically, Lucas sequences are obtained when $u_{0}=0$ (so, $c / d=-1$ ) or when $u_{0}=2, u_{1}=r$ (case in which $c=d=1$, so $c / d=1$ ). To essentially shift away from the situation of a primitive divisor theorem in disguise (so to avoid dealing with some binary recurrent sequence which is the subsequence of a Lucas sequence obtained, for example, by only selecting the terms of a Lucas sequence with indices in a fixed arithmetic progression), we make the assumption that

$$
c / d \text { and } \alpha / \beta \text { are multiplicatively independent. }
$$

Note that since $\alpha / \beta= \pm \alpha^{2}$, it follows that the above condition is equivalent to $c / d$ and $\alpha$ being multiplicatively independent, but the notation of (1.4) is more symmetric in the variables $c, d, \alpha, \beta$ which is why we keep the above formulation. Then we have the following theorem.

Theorem 1.1. Assume that $\left\{u_{n}\right\}_{n \geq 0}$ is a nondegenerate binary recurrent sequence of integers of characteristic equation $x^{2}-r x-s=0$ with $s \in\{ \pm 1\}$ whose roots are $\alpha$ and $\beta$. Assume that in the formula (1.1) the parameters $c / d$ and $\alpha / \beta$ satisfy (1.4). Then the Diophantine equation

$$
u_{n} u_{n+1} \cdots u_{n+k-1} \pm 1= \pm u_{m}^{2}
$$

has at most finitely many positive integer solutions $(k, m, n)$. Moreover, the solutions are effectively computable and satisfy

$$
m<10^{53}(Y+2)^{64},
$$

where

$$
Y:=\max \left\{\left|u_{0}\right|,\left|u_{1}\right|,|r|\right\}
$$

Our arguments can be applied to deal with the situation when the term \pm 1 is replaced by any nonzero integer, but the details are much more cumbersome.

As a toy example, we solve equation (1.5) in the concrete case when $\left\{u_{n}\right\}_{n \geq 0}$ is given by $u_{0}=-9, u_{1}=7$ and $u_{n+2}=u_{n+1}+u_{n}$ for all $n \geq 1$. The first few values of this sequence are

$$
-9,7,-2,5,3,8,11,19,30,49,79,128, \ldots
$$

Then $(\alpha, \beta)=((1+\sqrt{5}) / 2,(1-\sqrt{5}) / 2)$ and

$$
(c, d)=\left(\frac{23-9 \sqrt{5}}{2 \sqrt{5}},-\frac{23+9 \sqrt{5}}{2 \sqrt{5}}\right) \text {. }
$$


Condition (1.4) is satisfied since $c / d$ has trace $-467 / 31$ in $\mathbb{Q}(\sqrt{5})$, so in particular it is not an algebraic integer. Thus, $c / d$ and $\alpha / \beta$ are multiplicatively independent, otherwise $c / d$ will be a unit in $\mathbb{Q}(\sqrt{5})$, in particular an algebraic integer. We have the following result.

Theorem 1.2. For the sequence $\left\{u_{n}\right\}_{n \geq 0}$ given by $u_{1}=-9, u_{2}=7$ and $u_{n+2}=$ $u_{n+1}+u_{n}$ for all $n \geq 1$, the only solutions of equation (1.5) are

$$
\begin{aligned}
u_{3}-1 & =u_{2}^{2}, \\
u_{4}+1 & =u_{2}^{2}, \\
u_{5}+1 & =u_{4}^{2}, \\
u_{0} u_{1}-1 & =-u_{5}^{2}, \\
u_{2} u_{3}+1 & =-u_{4}^{2}, \\
u_{4} u_{5}+1 & =u_{3}^{2}, \\
u_{3} u_{4} u_{5}+1 & =u_{6}^{2} .
\end{aligned}
$$

\section{PRELiminaries}

Next let us notice that $u_{n} \neq 0$ for any $n \geq 0$. Indeed, if $u_{n}=0$ for some $n \geq$ 0 , then equation (1.1) shows that $c / d=-(\alpha / \beta)^{-n}$, contradicting (1.4). Thus, $\left|u_{n}\right| \geq 1$ for all $n \geq 1$. Next we justify that we may assume that $r>0$. Indeed, if $r=0$, then $\alpha / \beta=-1$, which is not allowed. If $r<0$, we may replace the pair $(r, s)$ by the pair $(-r, s)$. This has as effect replacing the pair $(\alpha, \beta)$ by $(-\alpha,-\beta)$. With (1.1), we get easily that, by keeping $u_{0}$ and changing the sign of $u_{1}$, we keep the values of $c$ and $d$ and, in particular, $u_{n}$ is then replaced by $(-1)^{n} u_{n}$. Hence, $\left|u_{n}\right|$ does not change under the above transformations. Since equation (1.5) is

$$
\left|u_{m}\right|^{2}=\left|u_{n} u_{n+1} \cdots u_{n+k} \pm 1\right|=\left|u_{n}\right|\left|u_{n+1}\right| \cdots\left|u_{n+k}\right| \pm 1,
$$

(for the last formula above we used the fact that $\left|u_{\ell}\right| \geq 1$ for all $\ell \geq 1$ ), it follows that we may indeed assume that $r>0$. Since $|\alpha|>1$, it follows that in fact $\alpha$ is positive, so

$$
(\alpha, \beta)=\left(\frac{r+\sqrt{\Delta}}{2}, \frac{r-\sqrt{\Delta}}{2}\right) .
$$

Further, if $c<0$, we then replace $(c, d)$ by $(-c,-d)$. This has as effect changing the sequence $\left\{u_{n}\right\}_{n \geq 1}$ to $\left\{-u_{n}\right\}_{n \geq 1}$, but as we already saw above, such transformation does not affect the solutions of (1.5). Hence, we assume that $c>0$. 
Below we record some lemmas.

Lemma 2.1. We have $\max \left\{\left|u_{0}\right|,\left|u_{1}\right|,\left|u_{2}\right|,\left|u_{3}\right|\right\}>1$.

As the referee pointed out, this lemma is a special case of Theorem 3 in [1]. We give a short proof here, the argument from [1] being quite involved.

Proof. Assume that $\left|u_{0}\right|=\left|u_{1}\right|=1$. Replacing $\left\{u_{n}\right\}_{n \geq 0}$ by $\left\{-u_{n}\right\}_{n \geq 0}$ if necessary, we may assume that $u_{0}=1$. Since $s= \pm 1$ we either have $s=u_{2}$ or $s=-u_{2}$. If $u_{2}= \pm 1$, we then get $\pm 1=u_{2}=r u_{1}+s u_{0}= \pm r+s$. If $s=u_{2}$, we get $r=0$, which is not allowed. So, $s=-u_{2}$ and $r=2 u_{1} u_{2}$. Since $r>0$, we first get that $r=2$, then that the characteristic equation must be $x^{2}-2 x-1=0$ (for the other option $x^{2}-2 x+1=0$ has a double root at $x=1$, which is not allowed), so $s=1=-u_{2}$ and $2=r=2 u_{1} u_{2}=-2 u_{1}$, so $u_{1}=-1$. Then $u_{3}=r u_{2}+s u_{1}=$ $-2-1=-3$, which gives us the desired conclusion.

Lemma 2.2. Let $p$ be any prime. Then $\left\{u_{n}\right\}_{n \geq 1}$ is periodic modulo $p$ with period at most $p^{2}-1$.

Proof. This is well-known.

We next need bounds on $\left|u_{n}\right|$ which are explicit. First, let us recall some terminology.

Let $\eta$ be an algebraic number of degree $\operatorname{deg}(\eta)$, whose minimal polynomial over the integers is

$$
g(x):=a_{0} \prod_{i=1}^{\operatorname{deg}(\eta)}\left(x-\eta^{(i)}\right) .
$$

We assume that $a_{0}>0$. The logarithmic height of $\eta$ is defined as

$$
h(\eta):=\frac{1}{\operatorname{deg}(\eta)}\left(\log a_{0}+\sum_{i=1}^{d} \log \max \left\{\left|\eta^{(i)}\right|, 1\right\}\right) .
$$

We put

$$
|\eta|=\max \left\{\left|\eta^{(i)}\right|: i=1, \ldots, \operatorname{deg}(\eta)\right\}
$$

In particular,

$$
\log |\eta|<\operatorname{deg}(\eta) h(\eta)
$$


We also put $\operatorname{den}(\eta)$ for the smallest positive integer $k$ such that $k \eta$ is an algebraic integer. Since $\operatorname{den}(\eta)$ divides $a_{0}$, it follows that

$$
\log \operatorname{den}(\eta) \leq \operatorname{deg}(\eta) h(\eta)
$$

Recall that

$$
Y:=\max \left\{r,\left|u_{0}\right|,\left|u_{1}\right|\right\} .
$$

Note that $Y \geq 2$, for if $Y=1$, then $r=\left|u_{0}\right|=\left|u_{1}\right|=1$, so $s=1$ and then $\left\{u_{n}\right\}_{n \geq 0}$ is, up to signs, some shift of the Fibonacci sequence, more precisely $\left\{u_{n}\right\}_{n \geq 0}$ is one of $\left\{F_{n+1}\right\}_{n \geq 0},\left\{-F_{n+1}\right\}_{n \geq 0},\left\{F_{n-2}\right\}_{n \geq 0}$ or $\left\{-F_{n-2}\right\}_{n \geq 0}$, all of which fail condition (1.4). In what follows, we write $\mathbb{K}:=\mathbb{Q}(\sqrt{\Delta})$.

The following results are similar to some results from [3].

Lemma 2.3. We have

$$
\max \{h(\alpha), h(\beta), h(\alpha / \beta), h(c), h(d), h(c / d)\}<6 \log (Y+1) .
$$

Proof. Since $\alpha+\beta=r \leq Y$ and $|\beta|<1$, we have that $1<\alpha<Y+1$. Thus,

$$
h(\alpha)=\frac{1}{2} \log \alpha<\frac{1}{2} \log (Y+1) .
$$

Since $\beta= \pm \alpha^{-1}$, we have that $h(\beta)=h(\alpha)$. Since $c=\left(u_{1}-u_{0} \beta\right) /(\alpha-\beta)$ and $c$ and $d$ are conjugates in $\mathbb{K}$, it follows that $h(c)=h(d)$. Further,

$$
\max \{|c|,|d|\} \leq \max \left\{\left|u_{0}\right|,\left|u_{1}\right|\right\}\left(\frac{\alpha+1}{\alpha-\beta}\right) \leq Y(Y+2)<(Y+1)^{2},
$$

where we used the fact that $\alpha-\beta=\sqrt{\Delta} \geq 1$ and $\alpha+1<Y+2$. Finally, it is easy to see that if $a_{0}$ is the leading coefficient of the minimal polynomial (over $\mathbb{Z})$ of $c$, then $a_{0} \mid(\alpha-\beta)^{2}$. Thus,

$$
a_{0} \leq(\alpha-\beta)^{2}=\Delta=r^{2}+4 s \leq r^{2}+4<(Y+1)^{2} .
$$

Putting all these facts together we get that

$$
\begin{aligned}
h(c)=h(d) & =\frac{1}{2}\left(\log a_{0}+\log \max \{1,|c|\}+\log \max \{1,|d|\}\right) \\
& <\frac{1}{2}\left(\log (Y+1)^{2}+2 \log (Y+1)^{2}\right)=3 \log (Y+1) .
\end{aligned}
$$

Finally,

$$
h(c / d) \leq h(c)+h(d)<6 \log (Y+1) .
$$


We put

$$
C_{0}:=6 \log (Y+1)+2
$$

Lemma 2.4. We have

$$
\left|u_{n}\right|<\alpha^{n+C_{0}}
$$

for all $n \geq 0$.

Proof. Using (1.1) and (1.3) we have

$$
\left|u_{n}\right| \leq \max \{|c|,|d|\}\left(\alpha^{n}+1\right) \leq 2 \alpha^{n}(Y+1)^{2}=\alpha^{n+\frac{\log 2}{\log \alpha}+\frac{2 \log (Y+1)}{\log \alpha}}<\alpha^{n+C_{0}},
$$

where we used the fact that $\alpha \geq(1+\sqrt{5}) / 2$.

Lemma 2.5. If $n \geq C_{0}$, then

$$
\left|u_{n}\right|>\alpha^{n-C_{0}}
$$

Proof. We write by (1.1)

$$
\left|u_{n}\right|=|c| \alpha^{n}\left|1+\frac{d}{c}\left(\frac{\beta}{\alpha}\right)^{n}\right| .
$$

Note that

$$
2\left|\frac{d}{c}\right|=2\left(\frac{\left|u_{1}-\alpha u_{0}\right|^{2}}{\left|N_{\mathbf{K}}\left(u_{1}-\alpha u_{0}\right)\right|}\right) \leq 2 \max \left\{\left|u_{0}\right|,\left|u_{1}\right|\right\}^{2}(1+\alpha)^{2}<2(Y+2)^{4} .
$$

Here, $N_{\mathbb{K}}(\bullet)$ is the norm from $\mathbb{K}$ to $\mathbb{Q}$. In the above (and later throughout the paper), we used that $u_{1}-\alpha u_{0}$ is a nonzero algebraic integer in $\mathbb{K}$, therefore $\left|N_{\mathbb{K}}\left(u_{1}-\alpha u_{0}\right)\right| \geq 1$. But $\alpha \geq(1+\sqrt{5}) / 2>e^{1 / 3}$ and $n \geq C_{0}$, so the above inequality implies

$$
\left|\frac{\alpha}{\beta}\right|^{n}=\alpha^{2 n}>e^{2 n / 3}>e^{4 \log (Y+2)+4 / 3}>2(Y+2)^{4}>2\left|\frac{d}{c}\right|,
$$

showing that

$$
\left|\frac{d}{c}\left(\frac{\beta}{\alpha}\right)^{n}\right|<\frac{1}{2}
$$

It follows, from (2.8), (2.7) and the absolute value inequality, that

$$
\left|u_{n}\right|>\frac{|c| \alpha^{n}}{2}=\frac{\left|N_{\mathbb{K}}\left(u_{1}-\alpha u_{0}\right)\right| \alpha^{n}}{2(\alpha-\beta)\left|u_{1}-\alpha u_{0}\right|} \geq \frac{\alpha^{n}}{2(\alpha+1)^{2} Y} \geq \frac{\alpha^{n-4}}{(Y+2)^{2}}>\alpha^{n-C_{0}},
$$

where for the last inequality we used $\alpha>e^{1 / 3}, 2 Y<(Y+2)^{2}, \alpha^{2} \geq \alpha+1$. 


\section{ON THE NUMBERS $u_{m} \pm 1$ AND $u_{m} \pm i$}

Rewriting equation (1.5) as

$$
u_{n} u_{n+1} \cdots u_{n+k-1}= \pm\left(u_{m}^{2} \pm 1\right)= \pm\left(u_{m}+\varepsilon\right)\left(u_{m}-\varepsilon\right) \quad \text { with } \quad \varepsilon \in\{1, i\}
$$

it follows that it makes sense to study the expressions $u_{m}-\varepsilon$ for $\varepsilon \in\{ \pm 1, \pm i\}$. Using (1.1), we get

$$
u_{m}-\varepsilon=c \alpha^{-m}\left(\alpha^{2 m}-\frac{\varepsilon}{c} \alpha^{m}+\frac{(-s)^{m} d}{c}\right)=c \alpha^{-m}\left(\alpha^{m}-z_{1}^{(m, \varepsilon)}\right)\left(\alpha^{m}-z_{2}^{(m, \varepsilon)}\right)
$$

where

$$
z_{1,2}^{(m, \varepsilon)}=\frac{\frac{\varepsilon}{c} \pm \sqrt{\left(\frac{\varepsilon}{c}\right)^{2}-\frac{4(-s)^{m} d}{c}}}{2}=\frac{\varepsilon \pm \sqrt{\varepsilon^{2}-4(-s)^{m} c d}}{2 c} .
$$

In the above calculation, we used that $c>0$. Note that also that expressions (3.1) and (1.3) show that

$$
z_{1,2}^{(m, \varepsilon)}=\frac{\varepsilon \sqrt{\Delta} \pm \sqrt{\varepsilon^{2} \Delta-4(-s)^{m} N_{\mathbb{K}}\left(u_{1}-\alpha u_{0}\right)}}{2 \sqrt{\Delta} c} .
$$

In particular, the number $\left(2 \sqrt{\Delta} c z_{1,2}^{(m, \varepsilon)}\right)^{2}$ is an algebraic integer of degree at most 2. For simplicity, we write $\zeta$ for any of $\zeta_{1,2}^{(m, \varepsilon)}$. Let us give some estimates on $h(\zeta)$. We start with the following lemma.

Lemma 3.1. If $\zeta \in \mathbb{Q}$, then $c+d= \pm 1$ and $\zeta \in\{ \pm 1\}$.

Proof. Assume $\zeta \in \mathbb{Q}$. We have either

$$
\zeta^{2}-\frac{\varepsilon}{c} \zeta+\frac{(-s)^{m} d}{c}=0
$$

or

$$
\zeta^{2}-\frac{\varepsilon}{d} \zeta+\frac{(-s)^{m} c}{d}=0
$$

Assume first that $\varepsilon \in\{ \pm i\}$. Then applying the complex conjugation we conclude that if $\zeta$ satisfies either of (3.3) or (3.4), then it will also satisfy the same equation with $\varepsilon$ replaced by $-\varepsilon$. Subtracting the two resulting relations, we get that either $2 \varepsilon \zeta / c=0$ (or $2 \varepsilon \zeta / d=0$ ), so $\zeta=0$, implying $d / c=0$, a contradiction. Thus, $\varepsilon \in\{ \pm 1\}$. Using the conjugation from $\mathbb{K}$ we conclude that $\zeta$ satisfies both (3.3) 
and (3.4). With the substitution $c_{1}:=-\varepsilon c$ and $d_{1}:=-\varepsilon(-s)^{m} d$, we get that the two quadratics

$$
X^{2}+\frac{1}{c_{1}} X+\frac{d_{1}}{c_{1}} \quad \text { and } \quad X^{2}+\frac{1}{d_{1}} X+\frac{c_{1}}{d_{1}}
$$

have a common root. Their resultant is

$$
\frac{\left(c_{1}-d_{1}\right)^{2}\left(\left(c_{1}+d_{1}\right)^{2}-1\right)}{\left(c_{1} d_{1}\right)^{2}} .
$$

Imposing that it is zero, we get that either $c_{1}=d_{1}$, or $c_{1}+d_{1}= \pm 1$. The first case leads to $c / d= \pm 1$, which is not possible because of condition (1.4). The second leads to either $c-d= \pm 1$ or $c+d= \pm 1$. The possibility $c-d= \pm 1$ together with $c+d=c+c^{\sigma} \in \mathbb{Q}$, leads to $c \in \mathbb{Q}$. Since $c$ and $d$ are conjugated, we get that $c / d=1$, which is again impossible. Thus, $c_{1}+d_{1}= \pm 1$ implies $c+d= \pm 1$, leading to $(-s)^{m}=1$. Putting now $\varepsilon_{1}:=\varepsilon(c+d)$, we get

$$
\begin{aligned}
u_{m}-\varepsilon & =\left(c \alpha^{m}+d \alpha^{-m}\right)-\varepsilon_{1}(c+d) \\
& =c\left(\alpha^{m}-\varepsilon_{1}\right)+d\left(\alpha^{-m}-\varepsilon_{1}\right) \\
& =c \alpha^{-m}\left(\alpha^{m}-\varepsilon_{1}\right)\left(\alpha^{m}-d \varepsilon_{1} / c\right)
\end{aligned}
$$

and we recognize our rational $\operatorname{root} \zeta=\varepsilon_{1} \in\{ \pm 1\}$.

Lemma 3.2. We have

$$
h(\zeta)<6 \log (Y+2)
$$

Proof. Since $\zeta$ is a root of a quadratic polynomial with coefficients in $\mathbb{K}(i)$, it follows that $\operatorname{deg}(\zeta) \leq 8$. Further, Lemma 3.1 shows that if $\zeta \in \mathbb{Q}$, then $\zeta= \pm 1$, so $h(\zeta)=0$. Thus, we may assume that $\zeta \notin \mathbb{Q}$, therefore $\operatorname{deg}(\zeta) \geq 2$. We use expression (3.2) for $\zeta$. Thus, the conjugates of $\zeta$ have similar formulas with $\varepsilon$ replaced eventually by $-\varepsilon$ or $\pm \bar{\varepsilon}$ and with $\sqrt{\Delta}$ replaced by $-\sqrt{\Delta}$. Further, $\sqrt{\Delta} c$ and $\sqrt{\Delta} d$ are conjugated quadratic integers, so their reciprocals in absolute value are at most

$$
\sqrt{\Delta} \max \{|c|,|d|\}=\max \left\{\left|u_{1}-\alpha u_{0}\right|,\left|u_{1}-\beta u_{0}\right|\right\} .
$$


Thus,

$$
\begin{aligned}
\zeta \zeta & \leq \frac{1}{2}\left(\sqrt{Y^{2}+4}+\sqrt{Y^{2}+4+4\left|N_{\mathbb{K}}\left(u_{1}-\alpha u_{0}\right)\right|}\right) \sqrt{\Delta} \max \{|c|,|d|\} \\
& \leq \frac{1}{2}\left(2 Y+\sqrt{Y^{2}+4+8 \max \left\{\left|u_{0}\right|,\left|u_{1}\right|\right\}^{2}(1+\alpha)}\right) \max \left\{\left|u_{1}\right|,\left|u_{0}\right|\right\}(1+\alpha) \\
& \leq \frac{1}{2}\left(2 Y+\sqrt{Y^{2}+4+8 Y^{2}(Y+2)}\right) Y(Y+2) \\
& \leq \frac{1}{2}\left(2 Y+(Y+2)^{3 / 2} \sqrt{8}\right)(Y+2)^{2}<2(Y+2)^{7 / 2} \leq(Y+2)^{4} .
\end{aligned}
$$

Also, we can see that $a_{0}$ divides $\Delta c d$, which is an integer. Its size is

$$
\left|N_{\mathbb{K}}\left(u_{1}-\alpha u_{0}\right)\right| \leq 2 \max \left\{\left|u_{1}\right|,\left|u_{0}\right|\right\}^{2}(1+\alpha)<2 Y^{2}(Y+2)<(Y+2)^{4} .
$$

Thus,

$$
\begin{aligned}
h(\zeta) & <\frac{1}{\operatorname{deg}(\zeta)}\left(\log (Y+2)^{4}+\operatorname{deg}(\zeta) \log (Y+2)^{4}\right) \\
& =4 \log (Y+2)\left(1+\frac{1}{\operatorname{deg}(\zeta)}\right) \leq 6 \log (Y+2)
\end{aligned}
$$

We need to understand whether $\zeta, c / d$ and $\alpha$ can be multiplicatively dependent. We have the following lemma.

Lemma 3.3. Assume that there are integers $(x, y, z)$ not all zero with with

$$
\zeta^{z}(c / d)^{y} \alpha^{x}=1 .
$$

Then there exist a nonzero integer vector $\left(x_{0}, y_{0}, z_{0}\right)$ and an integer $\lambda$ such that

$$
\zeta^{z_{0}}(c / d)^{y_{0}} \alpha^{x_{0}}= \pm 1, \quad \max \left\{\left|x_{0}\right|,\left|y_{0}\right|,\left|z_{0}\right|\right\}<250000(\log (Y+2))^{2},
$$

and

$$
(x, y, z)=\lambda\left(x_{0}, y_{0}, z_{0}\right)
$$

The proof of this lemma is based on the following result of Masser [7, Theorem $\mathbf{G}_{m}$ on page 253], which tells that the group of multiplicative relations of several non-zero algebraic numbers has bounded generators.

Proposition 3.4 (Masser). Let $\gamma_{1}, \ldots \gamma_{n}$ be non-zero algebraic numbers and let $\Gamma=\left\langle\gamma_{1}, \ldots \gamma_{n}\right\rangle$ the multiplicative group they generate. Further, let

$$
\eta=\min \{h(\gamma): \gamma \in \Gamma, h(\gamma)>0\}
$$


be the smallest positive height of an element of $\Gamma$, and $\omega$ the total number of roots of unity in the number field $\mathbb{Q}\left(\gamma_{1}, \ldots, \gamma_{n}\right)$. Then the subgroup of $\mathbb{Z}^{n}$ consisting of $\left(x_{1}, \ldots, x_{n}\right)$ such that $\gamma_{1}^{x_{1}} \cdots \gamma_{n}^{x_{n}}=1$ has a generating set consisting of elements satisfying

$$
\max \left\{\left|x_{1}\right|, \ldots,\left|x_{n}\right|\right\} \leq n^{n-1} \omega\left(\frac{h}{\eta}\right)^{n-1}
$$

where $h=\max \left\{h\left(\gamma_{1}\right), \ldots, h\left(\gamma_{n}\right)\right\}$.

Proof of Lemma 3.3. We will apply Masser's result to the numbers $\alpha^{2},(c / d)^{2}$ and $\zeta^{2}$. Note using (3.2) that

$$
\zeta^{2}=\left(\frac{\varepsilon \sqrt{\Delta} \pm \sqrt{\varepsilon^{2} \Delta-4(-s)^{m} N_{\mathbb{K}}\left(u_{1}-\alpha u_{0}\right)}}{2 \sqrt{\Delta} c}\right)^{2}=\frac{A+\sqrt{C}}{\Delta c^{2}} .
$$

for some integers $A$ and $C$. It follows that $\zeta^{2}$ is of degree at most 2 over the field $\mathbb{Q}(\sqrt{\Delta})$. In particular, $\alpha^{2},(c / d)^{2}$ and $\zeta^{2}$ generate a field of degree at most 4 , which implies that the parameter $\omega$ in Masser's result is bounded by 12 .

Furthermore, the smallest non-zero height of an algebraic number of degree at most 4 is that of the root of $X^{4}-X-1$, and this height is equal to

$$
\frac{1}{4} \log 1.38027756 \ldots>0.08
$$

(see [5, page 477]). Hence, $\eta>0.08$. Finally, Lemmas 2.3 and 3.2 imply that $h=12 \log (Y+2)$ would do.

Since $\alpha$ and $c / d$ are multiplicatively independent by (1.4), the group of integral vectors $(x, y, z)$ satisfying $\zeta^{2 z}(c / d)^{2 y} \alpha^{2 x}=1$ is of rank 1 . By the result of Masser, this group is generated by $\left(x_{0}, y_{0}, z_{0}\right) \in \mathbb{Z}^{3}$ satisfying

$$
\max \left\{\left|x_{0}\right|,\left|y_{0}\right|,\left|z_{0}\right|\right\} \leq 3^{2} \cdot 12 \cdot\left(\frac{12 \log (Y+2)}{0.08}\right)^{2}<250000(\log (Y+2))^{2} .
$$

We have clearly $\zeta^{z_{0}}(c / d)^{y_{0}} \alpha^{x_{0}}= \pm 1$, and our $(x, y, z)$ is an integral multiple of $\left(x_{0}, y_{0}, z_{0}\right)$.

4. ON THE GREATEST COMMON DIVISOR OF $\alpha^{m_{1}} \pm c / d$ AND $\alpha^{m_{2}}-\zeta_{1,2}^{(m, \varepsilon)}$

We keep our $\alpha$ and let $\zeta_{1}= \pm c / d$ and $\zeta_{2}=\zeta$ for some fixed $m$ and $\varepsilon$. Let $\mathbb{L}$ be some number field containing $\alpha, \zeta_{1}, \zeta_{2}$ of degree $D$. Put

$$
Z:=\max \left\{h(\alpha), h\left(\zeta_{1}\right), h\left(\zeta_{2}\right)\right\} \text {. }
$$


Note that $Z \leq 6 \log (Y+2)$. We need the following lemma.

Lemma 4.1. Let $m_{1}$ and $m_{2}$ be positive integers and I be an ideal of $\mathscr{O}_{\mathbb{L}}$ dividing both $\alpha^{m_{1}}-\zeta_{1}$ and $\alpha^{m_{2}}-\zeta_{2}$. Then putting $M:=\max \left\{m_{1}, m_{2}, 3\right\}$, one of the following holds:

(i) $N_{\mathbb{L}}(I)<\exp \left(6 D^{2} Z \sqrt{M}\right)$.

(ii) There exist integers $\left(x_{0}, y_{0}, z_{0}\right)$ not all 0 with

$$
\max \left\{\left|x_{0}\right|,\left|y_{0}\right|,\left|z_{0}\right|\right\} \leq 250000(\log (Y+2))^{2},
$$

such that

$$
z_{0} m_{1}+y_{0} m_{2}+x_{0}=0
$$

Proof. Write $\alpha^{m_{i}} \equiv \zeta_{i}(\bmod I)$ with $i=1,2$. There exist integers $\left(v_{1}, v_{2}\right)$ not both zero such that $\max \left\{\left|v_{1}\right|,\left|v_{2}\right|\right\} \leq \sqrt{M}$ and $\left|v_{1} m_{1}+v_{2} m_{2}\right| \leq 3 \sqrt{M}$ (see Lemma 1 in [4]). Up to replacing $\left(v_{1}, v_{2}\right)$ by $\left(-v_{1},-v_{2}\right)$, if needed, we may assume that $v_{1} m_{1}+v_{2} m_{2} \geq 0$. Exponentiating the above congruences to the powers $v_{1}$ (for $i=1$ ) and $v_{2}$ (for $i=2$ ) and multiplying the resulting congruences we get

$$
\alpha^{v_{1} m_{1}+v_{2} m_{2}} \equiv \zeta_{1}^{v_{1}} \zeta_{2}^{v_{2}} \quad(\bmod I) \text {. }
$$

Thus, $I$ divides the algebraic number

$$
\alpha^{v_{1} m_{1}+v_{2} m_{2}}-\zeta_{1}^{v_{1}} \zeta_{2}^{v_{2}}
$$

We now look at the above number. Assume first that the number shown at (4.2) is nonzero. Let $\Gamma$ be a common denominator of $\zeta_{1}^{ \pm 1}, \zeta_{2}^{ \pm 1}$. By inequality (2.2), it follows that

$$
\log \Gamma \leq D Z
$$

Putting $v:=2 \max \left\{\left|v_{1}\right|,\left|v_{2}\right|\right\}$, we get that

$$
I \mid \Gamma^{v}\left(\alpha^{v_{1} m_{1}+v_{2} m_{2}}-\zeta_{1}^{v_{1}} \zeta_{2}^{v_{2}}\right)=\Gamma^{v} \alpha^{v_{1} m_{1}+v_{2} m_{2}}-\Gamma^{v^{\prime}}\left(\Gamma \zeta_{1}^{\varepsilon_{1}}\right)^{\left|v_{1}\right|}\left(\Gamma \zeta_{2}^{\varepsilon_{2}}\right)^{\left|v_{2}\right|},
$$

where $\varepsilon_{i}=\operatorname{sign}\left(v_{i}\right)$ for $i=1,2$, and $v^{\prime}=v-v_{1}-v_{2} \geq 0$. The last number above is a nonzero algebraic integer in $\mathbb{L}$. Computing norms in $\mathbb{L}$, we get

$$
\begin{aligned}
\left|N_{\mathbb{L}}(I)\right| & \leq \prod_{i=1}^{D}\left|\Gamma^{v}\left(\alpha^{(i)}\right)^{v_{1} m_{1}+v_{2} m_{2}}-\Gamma^{v^{\prime}}\left(\Gamma \zeta_{1}^{(i)}\right)^{v_{1}}\left(\Gamma \zeta_{2}^{(i)}\right)^{v_{2}}\right| \\
& \leq 2^{D} \Gamma^{2 D \sqrt{M}}\left(\max \left\{\alpha^{3}, \zeta_{1}\left|\zeta_{2}\right|\right\}\right)^{D \sqrt{M}} \\
& \leq \exp \left(D \log 2+2 D \sqrt{M} \log \Gamma+D \sqrt{M} \max \left\{3 \log \alpha, \log \left(\left|\zeta_{1}\right| \zeta_{2} \mid\right)\right\}\right) \\
& <\exp \left(6 D^{2} Z \sqrt{M}\right) .
\end{aligned}
$$


In the above, we used in addition to estimates (2.1) and (2.2), the fact that $\log |\Gamma| \leq D Z$ together with the fact that $D Z \sqrt{M} \geq \sqrt{3} \log ((1+\sqrt{5}) / 2)>\log 2$. This is exactly (i).

Assume now that the number shown at (4.2) is zero. Then

$$
\zeta^{v_{2}}( \pm c / d)^{v_{1}} \alpha^{-\left(v_{1} m_{1}+v_{2} m_{2}\right)}=1
$$

Lemma 3.3 shows that there exist integers $\lambda \neq 0$ and $x_{0}, y_{0}, z_{0}$ not all three zero satisfying inequality (4.1) such that

$$
v_{2}=\lambda z_{0}, \quad v_{1}=\lambda y_{0}, \quad v_{1} m_{1}+v_{2} m_{2}=-\lambda x_{0} .
$$

Inserting the first two into the third we get

$$
y_{0} m_{1}+z_{0} m_{2}+x_{0}=0,
$$

which is (ii).

\section{THE PROOF OF THEOREM 1.1}

We split the proof in various steps. We assume that $m$ is sufficiently large (with respect to $Y$ ), where sufficiently large will be made explicit at each step.

\subsection{Bounding $n+k$ in terms of $m$.}

Lemma 5.1. If $m>3 C_{0}+3$, then $n+k<3 m$.

Proof. If $n+k<C_{0}+1$, then there is nothing to prove. Thus, assume that $n+k \geq$ $C_{0}+1$. In particular, $n+k>4$, so $\left|u_{n}\right| \cdots\left|u_{n+k-1}\right| \geq 2$ by Lemma 2.1. We then have, by Lemmas 2.5 and 2.4 , that

$$
\begin{aligned}
\alpha^{n+k-C_{0}-3} & <\frac{\alpha^{n+k-1-C_{0}}}{2} \leq \frac{\left|u_{n+k-1}\right|}{2} \leq\left|u_{n}\right|\left|u_{n+1}\right| \cdots\left|u_{n+k-1}\right|-1 \\
& \leq\left|u_{n} u_{n+1} \cdots u_{n+k-1} \pm 1\right|=\left|u_{m}\right|^{2}<\alpha^{2 m+2 C_{0}}
\end{aligned}
$$

so

$$
n+k<2 m+3 C_{0}+3<3 m \text {. }
$$


5.2. A dichotomy. Assume that $m>100 C_{0}$. We rewrite our equation as

$$
\begin{aligned}
\prod_{i=0}^{k-1}\left(c \alpha^{n+i}+d \beta^{n+i}\right) & =\prod_{i=0}^{k-1} u_{n+i}=u_{m}^{2} \pm 1=\left(u_{m}-\varepsilon\right)\left(u_{m}+\varepsilon\right) \\
& =c^{2} \alpha^{-2 m} \prod_{\substack{j=1,2 \\
\delta \in\{ \pm \varepsilon\}}}\left(\alpha^{m}-z_{j}^{(m, \delta)}\right) .
\end{aligned}
$$

We fix $m$ and $\varepsilon$ and work in the field $\mathbb{L}$ containing $\mathbb{K}$ and all four numbers $z_{1,2}^{(m, \pm \varepsilon)}$, which is of degree

$$
D \leq 16
$$

since it is contained in $\mathbb{Q}(\alpha, i)\left(z_{1}^{(m, \varepsilon)}, z_{1}^{(m,-\varepsilon)}\right)$ and each of $z_{1}^{(m, \pm \varepsilon)}$ is at most quadratic over $\mathbb{Q}(\sqrt{\Delta}, i)$. Letting

$$
Z:=\max \left\{h(\alpha), h(c / d), h\left(z_{1,2}^{(m, \pm \varepsilon)}\right)\right\}
$$

we get that

$$
Z \leq 6 \log (Y+2)
$$

by Lemmas 2.3 and 3.2 .

The condition $m>100 C_{0}$ insures that

$$
m>6 D Z \text {. }
$$

For $i \in\{0,1, \ldots, k-1\}, j \in\{1,2\}$ and $\delta \in\{ \pm \varepsilon\}$, we put

$$
I_{i, j}^{(m, \delta)}=\operatorname{gcd}\left(c \alpha^{n+i}+d \beta^{n+i}, \alpha^{m}-\zeta_{j}^{(m, \delta)}\right)
$$

as an ideal of $\mathscr{O}_{\mathbb{L}}$. We show that $c$ is invertible modulo $I_{i, j}^{(m, \delta)}$. For simplicity, let $I$ stand for this last ideal. Well, assume that it isn't. Then there exists a prime ideal $\pi$ appearing at a positive exponent in the factorization of the fractional ideal $c \mathscr{O}_{\mathbb{L}}$ such that $\pi$ also divides $I$. But then $\pi$ also divides $d$. In particular, letting $p$ be the prime number sitting above $\pi$, we get that $p$ divides $u_{n}$ for all $n \geq 1$. Reducing equation (1.5) modulo $p$, we get $\pm 1 \equiv 0(\bmod p)$, so (1.5) has no positive integer solution at all. Thus,

$$
I \mid \operatorname{gcd}\left(\alpha^{2(n+i)}-\zeta_{1}, \alpha^{m}-\zeta_{2}\right) \quad \text { where } \quad\left(\zeta_{1}, \zeta_{2}\right)=\left(-(-s)^{n+i} d / c, \zeta_{j}^{(m, \delta)}\right) .
$$

By Lemma 5.1, we can take

$$
M=6 m,
$$


and then we have that $\max \{2(n+i), m\}<M$ for all $i \in\{0,1, \ldots, k-1\}$. Lemma 4.1 applies with the parameters $D, Z, M$ bounded as in (5.1), (5.2) and (5.4), and we conclude that either:

Condition (i): The inequality

$$
N_{\mathbb{L}}(I)<\exp \left(6 \cdot D^{2} \cdot 6 \log (Y+2) \sqrt{6 m}\right)<\exp (2000 D \log (Y+2) \sqrt{m})
$$

holds true for all $I=I_{i, j}^{(m, \delta)}$ and all our choices for $i, j$ and $\delta$;

or

Condition (ii): There exists $i \in\{0, \ldots, k-1\}, j \in\{1,2\}, \delta \in\{ \pm \varepsilon\}$ and $\left(x_{0}, y_{0}, z_{0}\right) \in \mathbb{Z}^{3} \backslash\{(0,0,0)\}$ with

$$
\max \left\{\left|x_{0}\right|,\left|y_{0}\right|,\left|z_{0}\right|\right\}<250000(\log (Y+2))^{2},
$$

such that

$$
2(n+i) z_{0}+m y_{0}+x_{0}=0
$$

holds.

From now on we analyze each of the above situations (i) and (ii).

5.3. The case of Condition (i). From now on, we assume that Condition (i) of the previous Section 5.2 holds.

5.3.1. A lower bound for $k$ in terms of $m$.

Lemma 5.2. If $m>100 C_{0}$, then

$$
k>\sqrt{m} /(16000 \log (Y+2)) .
$$

Proof. In this case, we have

$$
u_{m}^{2} \pm 1=\operatorname{gcd}\left(u_{m}^{2} \pm 1, \prod_{i=0}^{k-1} u_{n+i}\right)
$$

The right-hand side divides

$$
\operatorname{gcd}\left(\prod_{i=0}^{k-1}\left(c \alpha^{n+i}+d \beta^{n+i}\right), c^{2} \alpha^{-2 m} \prod_{\substack{j \in\{1,2\} \\ \delta \in\{ \pm \varepsilon\}}}\left(\alpha^{m}-z_{i, j}^{(m, \delta)}\right)\right),
$$


which, in turn, divides $\prod_{i, j, \delta} I_{i, j}^{(m, \delta)}$. Taking norms in $\mathbb{L}$ and using (5.5), we get

$$
\left(u_{m}^{2} \pm 1\right)^{D} \leq \prod_{i, j, \delta} N_{\mathbb{L}}\left(I_{i, j}^{(m, \delta)}\right) \leq \exp (8000 D k \log (Y+2) \sqrt{m}),
$$

so

$$
u_{m}^{2} \pm 1<\exp (8000 k \log (Y+2) \sqrt{m}) .
$$

Using again Lemma 2.5 and the fact that $\alpha>e^{1 / 3}$, we get

$$
\begin{aligned}
\exp (m / 2) & <\alpha^{3 m / 2}<\alpha^{2 m-2 C_{0}-2}<\frac{\alpha^{2\left(m-C_{0}\right)}}{2}<\alpha^{2\left(m-C_{0}\right)} \pm 1<u_{m}^{2} \pm 1 \\
& <\exp (8000 k \log (Y+2) \sqrt{m}),
\end{aligned}
$$

giving

$$
k>\sqrt{m} /(16000 \log (Y+2)),
$$

which is what we wanted.

5.3.2. A small prime factor of $\prod_{i=0}^{k-1} u_{n+i}$ and its multiplicity. By Lemma 2.1, we have $\max \left\{\left|u_{0}\right|,\left|u_{1}\right|,\left|u_{2}\right|,\left|u_{3}\right|\right\}>1$. Let $p_{0}$ be the smallest prime factor of $u_{0} u_{1} u_{2} u_{3}$. Let us write an upper bound on it.

Since

$$
\left|u_{2}\right| \leq \max \left\{\left|u_{0}\right|,\left|u_{1}\right|\right\}(r+1) \leq(Y+1)^{2},
$$

and by a similar argument

$$
\left|u_{3}\right| \leq \max \left\{\left|u_{2}\right|,\left|u_{1}\right|\right\}(r+1) \leq(Y+1)^{3},
$$

it follows that

$$
p_{0} \leq(Y+1)^{3} .
$$

Let $\ell_{0}$ be the period of $\left\{u_{n}\right\}_{n \geq 0}$ modulo $p_{0}$. By Lemma 2.2,

$$
\ell_{0}<p_{0}^{2}<(Y+1)^{6}
$$

Since $p_{0}$ divides some term of the sequence $\left\{u_{n}\right\}_{n \geq 1}$ (more precisely, one of the first four), it follows that among $u_{n}, u_{n+1}, \ldots, u_{n+k-1}$, there are at least $\left\lfloor k / \ell_{0}\right\rfloor$ of such terms which are all multiples of $p_{0}$. Assuming $k \geq 2 \ell_{0}$, it follows that

$$
\operatorname{ord}_{p_{0}}\left(u_{n} u_{n+1} \cdots u_{n+k-1}\right) \geq\left\lfloor k / \ell_{0}\right\rfloor \geq k /\left(2 \ell_{0}\right) .
$$

Combining the last inequality above with (5.7), we obtain right away the following lemma. 
Lemma 5.3. Assume that $m>100 C_{0}$ and that

$$
m>2 \cdot 10^{9} \ell_{0}^{2}(\log (Y+2))^{2} .
$$

Then

$$
\operatorname{ord}_{p_{0}}\left(u_{n} u_{n+1} \cdots u_{n+k-1}\right) \geq \frac{\sqrt{m}}{32000 \ell_{0} \log (Y+2)}
$$

The condition (5.10) insures that $k>\sqrt{m} /(16000 \log (Y+2))>2 \ell_{0}$. Using (5.9), we arrive at the conclusion that (5.10) is always satisfied for some $p_{0}$ if $m>2 \cdot 10^{9}(Y+1)^{14}$ (and this condition implies automatically that $m>100 C_{0}$ ), and the lower bound from $(5.11)$ is at least $\sqrt{m} /\left(32000(Y+1)^{7}\right)$, but in practice better bounds are possible which is why we formulated Lemma 5.3 in terms of the parameter $\ell_{0}$.

5.3.3. A p-adic linear form. We recall a version of the $p$-adic linear form in logarithms due to Kunrui Yu. Let $\alpha_{1}, \ldots, \alpha_{l}$ be nonzero algebraic numbers in a field $\mathbb{M}$ of degree $D_{1}$. Let $p$ be a prime, $\pi$ a prime ideal of $\mathscr{O}_{\mathbb{M}}$ lying above $p$, having ramification $e$ and residual degree $f$. Let $b_{1}, \ldots, b_{l}$ be nonzero integers. Put

$$
B \geq \max \left\{\left|b_{1}\right|, \ldots,\left|b_{l}\right|, 3\right\}
$$

Let

$$
H_{j} \geq \max \left\{h\left(\alpha_{j}\right), \log p\right\} \quad \text { for all } \quad j=1, \ldots, l .
$$

Let

$$
\Lambda=\prod_{i=1}^{l} \alpha_{i}^{b_{i}}-1
$$

and assume that $\Lambda \neq 0$. The following result of Kunrui Yu [11] bounds the exponent of $\pi$ in the prime ideal factorization of $\Lambda$ inside $\mathscr{O}_{\mathbb{M}}$.

Lemma 5.4. With the above notations, we have

$$
\operatorname{ord}_{\pi}(\Lambda) \leq 19\left(20 \sqrt{l+1} D_{1}\right)^{2(l+1)} e^{l-1}\left(\frac{p^{f}}{(f \log p)^{2}}\right) \log \left(e^{5} l D_{1}\right) H_{1} \cdots H_{l} \log B .
$$


5.3.4. An upper bound on $k$. We let $\pi_{0}$ be any prime ideal dividing $p_{0}$ in $\mathscr{O}_{\mathbb{L}}$, where $\mathbb{L}$ is a field containing $\alpha$ and all $\zeta_{1,2}^{(m, \pm \varepsilon)}$. We are interested in an upper bound for

$$
\begin{aligned}
\operatorname{ord}_{\pi_{0}}\left(u_{m}^{2} \pm 1\right) & =\sum_{\substack{j \in\{1,2\} \\
\delta \in\{ \pm \varepsilon\}}} \operatorname{ord}_{\pi_{0}}\left(\alpha^{m}-z_{j}^{(m, \delta)}\right) \\
& \leq 4 \max \left\{\operatorname{ord}_{\pi_{0}}\left(\alpha^{m}-\zeta\right): \zeta \in\left\{z_{1,2}^{(m, \pm \varepsilon)}\right\}\right\} .
\end{aligned}
$$

Note that since $u_{m}^{2} \pm 1=\prod_{i=1}^{k-1} u_{n+i}$ is nonzero, it follows that $\alpha^{m}-\zeta \neq 0$ for all $\zeta \in\left\{z_{1,2}^{(m, \pm \varepsilon)}\right\}$. To bound the right-hand side of (5.13), we use Lemma 5.4. We take $l=2, \alpha_{1}=\alpha^{-1}, \alpha_{2}=\zeta, b_{1}=m, b_{2}=1$. We can take $\mathbb{M}:=\mathbb{K}(\zeta)$ which is of degree $D_{1} \leq 8$. By Lemma 2.3 and inequality (5.8), we can take

$$
H_{1}=H_{2}:=6 \log (Y+2),
$$

and the inequality (5.12) is satisfied for our situation. Since $e f \leq D_{1}$, we get

$$
\operatorname{ord}_{\pi_{0}}\left(u_{m}^{2} \pm 1\right) \leq 4 \cdot 19(20 \sqrt{3} 8)^{6} \cdot 6 \frac{p_{0}^{8}}{(\log p)^{2}} \log \left(16 e^{5}\right)(8 \log (Y+2))^{2} \log m,
$$

so

$$
\operatorname{ord}_{\pi_{0}}\left(u_{m}^{2} \pm 1\right)<1.5 \cdot 10^{20}\left(\frac{p_{0}^{8}}{\left(\log p_{0}\right)^{2}}\right)(\log (Y+2))^{2} \log m .
$$

Since clearly

$$
\operatorname{ord}_{\pi_{0}}\left(u_{m}^{2} \pm 1\right) \geq \operatorname{ord}_{p_{0}}\left(u_{m}^{2} \pm 1\right)=\operatorname{ord}_{p_{0}}\left(u_{n} u_{n+1} \cdots u_{n+k-1}\right),
$$

Lemma 5.3 implies the following result.

Lemma 5.5. Assume that $m>100 C_{0}$ and that

$$
m>2 \cdot 10^{9} \ell_{0}^{2}(\log (Y+2))^{2} .
$$

Then

$$
\frac{\sqrt{m}}{32000 \ell_{0} \log (Y+2)}<1.5 \cdot 10^{20}\left(\frac{p_{0}^{8}}{\left(\log p_{0}\right)^{2}}\right)(\log (Y+2))^{2} \log m
$$

At this point the theorem is proved since inequality (5.14) gives a bound on $m$. If we want a specific bound, we may assume that $m>3 \cdot 10^{8}(Y+1)^{6}$, and then, using the fact that $p_{0} \leq(Y+2)^{3}$ and the fact that the function $t \mapsto t^{8} /(\log t)^{2}$ is increasing for $t \geq 2$, we get that

$$
\frac{\sqrt{m}}{32000(Y+1)^{7}}<1.5 \cdot 10^{20}\left(\frac{(Y+2)^{24}}{\left(\log (Y+2)^{3}\right)^{2}}\right)(\log (Y+2))^{2} \log m,
$$


which gives

$$
\sqrt{m}<6 \cdot 10^{23}(Y+2)^{31} \log m=12 \cdot 10^{23}(Y+2)^{31} \log \sqrt{m} .
$$

It is known that if $x / \log x<A$ for some $A>3$, then $x<2 A \log A$. Applying this with $x:=\sqrt{m}$ and $A:=6 \cdot 10^{23}(Y+2)^{24}$, we get

$$
\begin{aligned}
\sqrt{m} & <2 \cdot 12 \cdot 10^{23}(Y+2)^{31}\left(\log \left(12 \cdot 10^{23}\right)+31 \log (Y+2)\right) \\
& <24 \cdot 71 \cdot 10^{23}(Y+2)^{31} \log (Y+2) \\
& <2 \cdot 10^{26}(Y+2)^{32}
\end{aligned}
$$

In the above, we used that

$$
\begin{aligned}
\log \left(12 \cdot 10^{23}\right)+31 \log (Y+2) & \leq\left(\frac{\log \left(12 \cdot 10^{23}\right)}{\log 4}+31\right) \log (Y+2) \\
& <71 \log (Y+2) .
\end{aligned}
$$

Hence,

$$
m<10^{53}(Y+2)^{64},
$$

which completes the proof in the case of Condition (i).

5.4. The case of Condition (ii). From now on, we work under the Condition (ii).

5.4.1. The case when $y_{0} z_{0}=0$. We look at the relation (5.6). If both $y_{0}=z_{0}=0$, then $x_{0}=0$, so $\left(x_{0}, y_{0}, z_{0}\right)=(0,0,0)$, which is a contradiction. If $z_{0}=0$, then $\left|y_{0}\right| \geq 1$, so

$$
m \leq\left|x_{0}\right| \leq 250000(\log (Y+2))^{2},
$$

which is a better inequality than (1.6). If $y_{0}=0$, then $\left|z_{0}\right| \geq 1$, so

$$
2(n+i)\left|z_{0}\right| \leq\left|x_{0}\right| \leq 250000(\log (Y+2))^{2},
$$

giving

$$
n<125000(\log (Y+2))^{2} .
$$

Assume that $m>100 C_{0}$. Then Lemmas 2.4 and 2.5 show that

$$
\alpha^{n k+\left(k(k-1) / 2+k C_{0}\right.}>\left|u_{n+1}\right| \cdots\left|u_{n+k}\right|-1 \geq u_{m}^{2} / 2 \geq \alpha^{2 m-2-2 C_{0}},
$$

so

$$
k^{2}+\left(2 n+2 C_{0}\right) k>2 n k+k(k-1)+2 k C_{0}>2\left(2 m-2-C_{0}\right)>2 m .
$$


The left-hand side above is less than $\left(k+1000 C_{0}^{3}\right)^{2}$ because

$$
\left(k+1000 C_{0}^{3}\right)^{2}>k^{2}+\left(2000 C_{0}^{3}\right) k,
$$

and

$$
2000 C_{0}^{3}>2 n+2 C_{0},
$$

an inequality implied by (5.15). We thus get

$$
k+2000 C_{0}^{3}>\sqrt{2 m} .
$$

Assuming that

$$
m>10^{13}(\log (Y+2))^{6}
$$

we have that

$$
k>\sqrt{2 m}-2000 C_{0}^{3}>\sqrt{m} .
$$

We are now in the conditions of Lemma 5.2, and from now on the rest of the proof from the preceding case applies and leads to the desired inequality (1.6) on $m$.

From now on, we assume that

$$
y_{0} z_{0} \neq 0
$$

5.4.2. Bounding $k$. Since $y_{0} z_{0} \neq 0$, we have

$$
m \leq m\left|y_{0}\right| \leq 2(n+k)\left|z_{0}\right|+\left|x_{0}\right|<10^{6}(\log (Y+2))^{2}(n+k+1) .
$$

Thus, we may assume that $n+k$ is large. Assuming that $n+k>100 C_{0}$, we get, using again Lemmas 2.4 and 2.5 , that

$$
\left|u_{n}\right| \cdots\left|u_{n+k-1}\right| \geq\left|u_{n+\lfloor k / 2\rfloor}\right|\left|u_{n+\lfloor k / 2\rfloor+1}\right| \cdots\left|u_{n+k-1}\right| \geq \alpha^{(k / 2)\left(n+\lfloor k / 2\rfloor-C_{0}\right)},
$$

while

$$
u_{m}^{2} \pm 1 \leq 2 u_{m}^{2} \leq \alpha^{2 m+2+2 C_{0}} .
$$

We thus get, assuming that $m>100 C_{0}$, that

$$
(k / 2)\left(n+\lfloor k / 2\rfloor-C_{0}\right)<2 m+2+2 C_{0}<3 m<3 \cdot 10^{6}(\log (Y+2))^{2}(n+k+1) .
$$

Since $n+k>100 C_{0}$, we get that $n+\lfloor k / 2\rfloor-C_{0}>(n+k+1) / 3$, so the above inequality gives

$$
k<2 \cdot 10^{7}(\log (Y+2))^{2} .
$$

We record this as a lemma. 
Lemma 5.6. Assuming

$$
m>100 C_{0},
$$

we have that

$$
m<10^{6}(\log (Y+2))^{2}(n+k+1) .
$$

Furthermore, if also

$$
n+k>100 C_{0}
$$

then

$$
k<2 \cdot 10^{7}(\log (Y+2))^{2} .
$$

5.4.3. A linear form in archimedian logarithms. From now on, we assume that both (5.19) and (5.21) hold true. We may assume it, because if (5.19) is not true then we have an estimate for $m$ much stronger than (1.6). And if (5.19) is true but (5.21) is not then (5.20) again implies a bound much sharper than (1.6). As a consequence of the lemma above, we have (5.20) and (5.22).

We may also assume that $n$ is large; precisely, that

$$
n>100 \log (Y+2) \text {. }
$$

Indeed, if this is not true, then (5.20) and (5.22) again imply a much sharper bound than (1.6).

We look at

$$
u_{n+i}=c \alpha^{n+i}\left(1+\frac{(-s)^{n+i} d}{c \alpha^{2 n+2 i}}\right):=c \alpha^{n+i}\left(1+\zeta_{n, i}\right)
$$

Note that

$$
\left|\zeta_{n, i}\right|=\left|\frac{d / c}{\alpha^{2 n+2 i}}\right|<\frac{1}{\alpha^{n}}
$$

provided $\alpha^{n}>|d / c|$, which holds whenever (5.23) is true, because

$$
\log |d / c| \leq 2 h(c / d) \leq 12 \log (Y+2)
$$

(by Lemma 2.3), while also $\alpha \geq(1+\sqrt{5}) / 2$. Thus, equation (1.5) is

$$
c^{k} \alpha^{k n+k(k-1) / 2} \prod_{i=0}^{n}\left(1+\zeta_{n, i}\right) \pm 1=c^{2} \alpha^{2 m}\left(1+\zeta_{m, 0}\right)^{2} .
$$


If $|x|<1 / 2$, then $|\log (1+x)|<2|x|$. Thus,

$$
\log \prod_{i=0}^{k-1}\left(1+\zeta_{n, i}\right) \leq 2 \sum_{i=0}^{k-1}\left|\zeta_{n, i}\right|<\frac{2 k}{\alpha^{n}}<\frac{1}{\alpha^{n / 2}},
$$

where the last inequality holds provided that

$$
\alpha^{n / 2}>4 \cdot 10^{7}(\log (Y+2))^{2},
$$

because the right-hand side above is an upper bound for $2 k$ by Lemma 5.6. The last inequality above is certainly satisfied whenever (5.23) holds. Since $\alpha^{n / 2}>2$, it follows that

$$
\left|\prod_{i=0}^{k-1}\left(1+\zeta_{n, i}\right)-1\right|<\exp \left(\frac{1}{\alpha^{n / 2}}\right)-1<\frac{2}{\alpha^{n / 2}} .
$$

Similarly,

$$
\left|\left(1+\zeta_{m, 0}\right)^{2}-1\right|<\frac{2}{\alpha^{m / 2}}
$$

Thus, we may write

$$
u_{n} \cdots u_{n+k-1}=c^{k} \alpha^{n k+k(k-1) / 2}\left(1+\eta_{n, k}\right) \quad \text { and } \quad u_{m}^{2}=c^{2} \alpha^{2 m}\left(1+\eta_{m}\right),
$$

where

$$
\left|\eta_{n, k}\right|<\frac{2}{\alpha^{n / 2}} \quad \text { and } \quad\left|\eta_{m}\right|<\frac{2}{\alpha^{m / 2}} .
$$

Thus, equation (1.5) is

$$
c^{k} \alpha^{n k+k(k-1) / 2}\left(1+\eta_{n, k}\right) \pm 1=c^{2} \alpha^{2 m}\left(1+\eta_{m}\right),
$$

or

$$
c^{k-2} \alpha^{n k+k(k-1) / 2-2 m}\left(1+\eta_{n, k}\right)=\left(1+\eta_{m}\right) \mp \frac{1}{c^{2} \alpha^{2 m}} .
$$

We have

$$
\frac{1}{2}<\left|1+\eta_{n, k}\right|<\frac{3}{2}, \quad \frac{1}{2}<\left|1+\eta_{m}\right|<\frac{3}{2}, \quad \frac{1}{c^{2} \alpha^{2 m}}<\frac{1}{\alpha^{m}}<\frac{1}{4} .
$$

Here we used the inequality

$$
c^{2} \alpha^{m} \geq 1
$$

which is true because $\log \left(c^{2}\right) \geq-4 h(c) \geq-4 C_{0}$ (by Lemma 2.3) and on the other hand $\log \left(\alpha^{m}\right) \geq 100 C_{0} \log \frac{1+\sqrt{5}}{2}>4 C_{0}$ by (5.19). Using (5.24), we deduce

$$
\frac{1}{6}<c^{k-2} \alpha^{n k+k(k-1) / 2-2 m}<4 \text {. }
$$


Hence,

$$
\begin{aligned}
\left|c^{k-2} \alpha^{n k+k(k-1) / 2-2 m}-1\right| & <\frac{1}{c^{2} \alpha^{2 m}}+\left|\eta_{m}\right|+c^{k-2} \alpha^{n k+k(k-1) / 2-2 m}\left|\eta_{n, k}\right| \\
& <\frac{1}{\alpha^{m}}+\frac{2}{\alpha^{m / 2}}+\frac{8}{\alpha^{n / 2}} \\
& <\frac{11}{\alpha^{\min \{m / 2, n / 2\}}} .
\end{aligned}
$$

We want to estimate the left-hand side from below using the theory of linear forms in logarithms. But we need to ensure that the left-hand side is nonzero. It is not possible that $(k-2, n k+k(k-1) / 2,2 m)=(0,0)$, since this leads to $k=2$ and $2 n+1=2 m$, which is impossible. Thus, if the left-hand side of (5.25) is 0 , then $c$ and $\alpha$ are multiplicatively dependent. But since $d$ is conjugate to $c$ and $\beta= \pm \alpha^{-1}$ is conjugate to $\alpha$, it follows that also $d$ and $\alpha$ are multiplicatively dependent and therefore $c / d$ and $\alpha$ are multiplicatively dependent, which is not possible.

The following result is (a slightly weaker version of) Corollary 2.3 from Matveev [8].

Theorem 5.7. Let $\mathbb{L}$ be a number field of degree D over $\mathbb{Q}, \zeta_{1}, \ldots, \zeta_{t}$ be positive real numbers of $\mathbb{L}$, and $b_{1}, \ldots, b_{t}$ be integers. Put

$$
\Lambda:=\zeta_{1}^{b_{1}} \cdots \zeta_{t}^{b_{t}}-1 \text {. }
$$

Let $A_{1}, \ldots, A_{t}, B$ be real numbers such that

$$
\begin{aligned}
A_{i} & \geq \max \left\{D h\left(\zeta_{i}\right),\left|\log \zeta_{i}\right|, 0.16\right\}, \quad i=1, \ldots, t, \\
B & \geq \max \left\{\left|b_{1}\right|, \ldots,\left|b_{t}\right|\right\} .
\end{aligned}
$$

Then, assuming that $\Lambda \neq 0$, we have

$$
|\Lambda|>\exp \left(-1.4 \cdot 30^{t+3} \cdot t^{4.5} \cdot D^{2}(1+\log D)(1+\log B) A_{1} \cdots A_{t}\right) .
$$

For our application, we take $t=2, \zeta_{1}:=c, \zeta_{2}:=\alpha, b_{1}:=k-2, b_{2}:=$ $n k+k(k-1) / 2-2 m$. We can take $\mathbb{L}=\mathbb{Q}(\alpha)$; in particular, $D \leq 2$. As for the numbers $A_{1}, A_{2}$, we can take

$$
A_{1}=A_{2}=12 \log (Y+2) .
$$

We take $B=\max \left\{m, n k+k^{2}\right\}$. Then

$$
\log |\Lambda|>-1.4 \cdot 30^{5} \cdot 2^{4.5} \cdot 2^{2}(1+\log 2)(1+\log B)(12 \log (Y+2))^{2} .
$$


Thus,

$$
\log |\Lambda|>-10^{12}(\log (Y+2))^{2}(1+\log B) .
$$

Comparing this with (5.25), we get

$$
\min \{n / 2, m / 2\} \log \alpha-\log 11<10^{12}(\log (Y+2))^{2}(1+\log B) .
$$

We then get that

$$
\min \{n, m\} \leq 5 \cdot 10^{12}(\log (Y+2))^{2}(1+\log B) .
$$

We may assume that $n+k+1>2 \cdot 10^{6}(\log (Y+2))^{2}$, otherwise Lemma 5.6 gives us

$$
m<4 \cdot 10^{12}(\log (Y+2))^{4},
$$

which is better than the inequality (1.6). Then Lemma 5.6 shows that

$$
m<(n+k+1)^{2} \text { therefore } \log B \leq 2 \log (n+k+1) .
$$

Thus,

$$
\begin{aligned}
\min \{n, m\} & \leq 5 \cdot 10^{12}(\log (Y+2))^{2}(1+2 \log (n+k+1)) \\
& \leq 1.5 \cdot 10^{13}(\log (Y+2))^{2} \log (n+k+1)
\end{aligned}
$$

Assume first that $m \leq n$. By Lemma 5.1, we get

$$
n+k+1 \leq 3 m+1<4 m
$$

therefore with $x=4 m$, inequality (5.27) implies

$$
\left.x<6 \cdot 10^{13}(\log Y+2)\right)^{2} \log x .
$$

With $A=6 \cdot 10^{13}(\log (Y+2))^{2}$, we have $x<A \log x$, an inequality which implies that $x<2 A \log A$. Thus,

$$
\begin{aligned}
4 m & <12 \cdot 10^{13}\left(\log ((Y+2))^{2}\left(\log \left(6 \cdot 10^{13}\right)+2 \log \log (Y+2)\right)\right. \\
& <12 \cdot 34 \cdot 10^{13}(\log (Y+2))^{3}
\end{aligned}
$$

giving

$$
m<10^{16}\left(\log ((Y+2))^{3},\right.
$$

which is better than estimate (1.6).

Assume next that $n \leq m$. Then inequality (5.27) shows that

$$
n \leq 1.5 \cdot 10^{13}(\log (Y+2))^{2} \log (n+k+1) .
$$


With (5.22) we get

$$
\begin{aligned}
n+k+1 & <1.5 \cdot 10^{13}(\log (Y+2))^{2} \log (n+k+1) \\
& +\log \left(2 \cdot 10^{6}(\log (Y+2))^{3}\right)+1 \\
& <2 \cdot 10^{13}(\log (Y+2))^{3} \log (n+k+1) .
\end{aligned}
$$

As we said, for $A>2$ the inequality $x<A \log x$ implies $x<2 A \log A$. Taking $A=2 \cdot 10^{13}(\log (Y+2))^{2}$ and $x:=n+k+1$, we get

$$
\begin{aligned}
n+k+1 & <4 \cdot 10^{13}(\log (Y+2))^{2}(13 \log 10+2 \log \log (Y+2)) \\
& <2 \cdot 10^{15}(\log (Y+2))^{3}
\end{aligned}
$$

which together with (5.20) gives

$$
m<2 \cdot 10^{22}(\log (Y+2))^{5},
$$

which is better than the inequality (1.6). This finishes the proof of the theorem.

\section{THE PROOF OF THEOREM 1.2}

Plugging in our values, we get $Y=9$, so $m<10^{53} \cdot 11^{64}<10^{120}$. The period of $\left\{u_{m}\right\}_{m \geq 0}$ modulo 11 is 10 and $u_{6} \equiv 0(\bmod 11)$. We show that $k \leq 9$. For if not, then $[n, n+k-1]$ contains a number $n+i$ congruent to 6 modulo 10 so $11 \mid u_{n} \cdots u_{n+k-1}$. We get that

$$
u_{m}^{2} \equiv \pm 1 \quad(\bmod 11) .
$$

The congruence $u_{m}^{2} \equiv-1(\bmod 11)$ is not possible since 11 is a prime congruent to 3 modulo 4 (so, -1 is not a quadratic residue modulo 11). The congruence $u_{m}^{2} \equiv 1(\bmod 11)$ implies $u_{m} \equiv \pm 1(\bmod 11)$. However, the first 10 values of $\left\{u_{n}\right\}_{n \geq 1}$ modulo 11 are

$$
2,7,9,5,3,8,0,8,8,5
$$

none of which is $\pm 1(\bmod 11)$, so by periodicity we deduce that there is no positive integer $m$ such that $u_{m} \equiv \pm 1(\bmod 11)$. So, indeed $k \leq 9$.

We next show that either $n \leq 43$ or $m \leq 18$. Assume this is not so. For a positive integer $\ell$, we have

$$
u_{\ell}=c \alpha^{\ell}\left(1+\frac{(-1)^{\ell} d}{c \alpha^{2 \ell}}\right)
$$


Further,

$$
\left|\frac{d}{c}\right|=\frac{23+9 \sqrt{5}}{23-9 \sqrt{5}}<15 .
$$

In formula (6.1), we give $\ell$ the values $n+i$ for $i=0,1, \ldots, k-1$ and multiply the resulting expressions to get that

$$
\begin{aligned}
u_{n} \cdots u_{n+k-1} & =c^{k} \alpha^{n+(n+1)+\cdots+(n+k-1)} \prod_{i=0}^{k-1}\left(1+\frac{(-1)^{n+i} d}{c \alpha^{2 n+2 i}}\right) \\
& =c^{k} \alpha^{n k+k(k-1) / 2}\left(1+\zeta_{n, k}\right),
\end{aligned}
$$

where trivially

$$
\left|\zeta_{n, k}\right|<2^{k} \frac{|d / c|^{k}}{\alpha^{2 n}}<\frac{30^{9}}{\alpha^{2 n}}<\frac{1}{\alpha^{2 n-64}}
$$

Similarly,

$$
u_{m}^{2}=c^{2} \alpha^{2 m}\left(1+\frac{(-1)^{m} d / c}{\alpha^{2 m}}\right)^{2}=c^{2} \alpha^{2 m}\left(1+\zeta_{m}\right)
$$

where

$$
\left|\zeta_{m}\right|<\frac{3|d / c|^{2}}{\alpha^{2 m}}<\frac{775}{\alpha^{2 m}}<\frac{1}{\alpha^{2 m-14}}
$$

Further, we use the fact that

$$
\alpha^{\ell-2}<u_{\ell}<\alpha^{\ell-1} \quad \text { for all } \quad \ell \geq 4,
$$

which is easy to check by induction on $\ell$. Since

$$
u_{n} u_{n+1} \cdots u_{n+k-1} u_{m}^{-2}-1= \pm \frac{1}{u_{m}^{2}}
$$

we use (6.3) and (6.5) to get that

$$
c^{k-2} \alpha^{n k+k(k-1) / 2-2 m}\left(\frac{1+\zeta_{n . k}}{1+\zeta_{m}}\right)-1= \pm \frac{1}{u_{m}^{2}},
$$

which can be rewritten as

$$
c^{k-2} \alpha^{n k+k(k-1) / 2-2 m}-1= \pm \frac{\left(1+\zeta_{m}\right)}{\left(1+\zeta_{n, k}\right) u_{m}^{2}}+\frac{\zeta_{m}}{\left(1+\zeta_{n, k}\right)}-\frac{\zeta_{n, k}}{1+\zeta_{n, k}} .
$$

Taking absolute values and using estimates (6.4), (6.6) and (6.7) together with the fact that $\max \left\{\left|\zeta_{m}\right|,\left|\zeta_{n, k}\right|\right\}<1 / 2$ in our range of variables $n \geq 44$ and $m \geq 19$, 
we get

$$
\begin{aligned}
\left|c^{k-2} \alpha^{n k+k(k-1) / 2-2 m}-1\right| & \leq\left(\frac{3 / 2}{1 / 2}\right) \frac{1}{\alpha^{2(m-2)}}+\left(\frac{1}{1 / 2}\right) \frac{1}{\alpha^{2 m-14}} \\
& +\left(\frac{1}{1 / 2}\right) \frac{1}{\alpha^{2 n-64}} \\
& <\frac{7}{\alpha^{\min \{2 n-64,2 m-14\}}} \\
& <\frac{1}{\alpha^{\min \{2 n-69,2 m-19\}}} .
\end{aligned}
$$

Since we are assuming that $n \geq 44$ and $m \geq 19$, the right-hand side above is $<1 / 2$. It is known that if $\left|e^{x}-1\right|<y$ with $y<1 / 2$, then $|x|<2 y$. Applying this to our situation, we get

$$
\begin{aligned}
|(k-2) \log c-(k n+k(k-1) / 2-2 m) \log \alpha| & <\frac{2}{\alpha^{\min \{2 n-69,2 m-19\}}} \\
& <\frac{1}{\alpha^{\min \{2 n-71,2 m-21\}}}
\end{aligned}
$$

If $k=2$, we then get that

$$
|2 n+1-2 m| \log \alpha<\frac{1}{\alpha^{\min \{2 n-71,2 m-21\}}} .
$$

Since $2 n+1 \neq 2 m$, the left-hand side is at least $\log \alpha>0.48$, and, for $n \geq 44$ and $m \geq 19$, the right-hand side is at most $\alpha^{-17}<0.0003$, a contradiction. Hence, $k \neq 2$.

Dividing (6.8) by $(k-2) \log \alpha$, we get

$$
\begin{aligned}
\left|\frac{k n+k(k-1) / 2-2 m}{k-2}-\frac{\log c}{\log \alpha}\right| & <\frac{1}{|k-2|(\log \alpha) \alpha^{\min \{2 n-71,2 m-21\}}} \\
& <\frac{1}{\alpha^{\min \{2 n-73,2 m-23\}}} .
\end{aligned}
$$

The continued fraction of $\log c / \log \alpha$ is $[-1,12,5, \ldots]$ with the second convergent being $-11 / 12$. Since $|\log c / \log \alpha+11 / 12|>0.001$, and since $|k-2| \leq 7$ (because $k \leq 9$ ), we infer, from the properties of the continued fractions, that

$$
0.001<\frac{1}{\alpha^{\min \{2 n-73,2 m-23\}}},
$$

giving either $n<44$ or $m<19$, which is what we wanted. 
If $m \geq 19$, then $n \leq 43$, so

$$
\alpha^{2 m-6} \leq \frac{\alpha^{2(m-2)}}{2} \leq \frac{u_{m}^{2}}{2} \leq u_{m}^{2} \pm 1=u_{n} \cdots u_{n+k-1} \leq u_{n+8}^{9} \leq u_{51}^{9}<\alpha^{450},
$$

giving $m<228$. So, in all cases $m<228$. As for $n$, we either have $n<44$, or $n \geq 44$. But if $n \geq 44$, then $m \leq 18$. Since $\alpha^{n-2}<u_{n} \leq u_{m}^{2}+1<2 u_{m}^{2}<\alpha^{2 m} \leq$ $\alpha^{36}$, we get that $n<38$, contradicting that $n \geq 44$.

So, all solutions have $k \leq 9, m \leq 227$, and $n \leq 43$. A quick computation now confirms that the solutions listed in the statement of Theorem 1.2 are the only ones.

\section{ACKNOWLEDGEMENTS}

We thank the referee for careful reading and detecting a flaw in the initial version. We also thank Karim Belabas and Michael Mossinghoff for helpful suggestions.

\section{REFERENCES}

[1] F. Beukers, The multiplicity of binary recurrences, Compositio Math. 40: 251-267, 1980.

[2] J. J. Bravo, T. Komatsu and F. Luca, On the distance between products of consecutive Fibonacci numbers and powers of Fibonacci numbers, Indag. Math., 24:181-198, 2013.

[3] S. Guzman and F. Luca, Linear combinations of factorials and $S$-units in a Binary Recurrence Sequence, Ann. Math. Québec, 38: 169-188, 2014.

[4] T. Komatsu, F. Luca and Y. Tachiya, On the multiplicative order of $F_{n+1} / F_{n}$ modulo $F_{m}$, in "Proc. of the Integers Conference 2011", Integers 12: A8, 2012/2013.

[5] D. H. Lehmer, Factorization of Certain Cyclotomic Functions, Ann. Math. II 34: 461-479, 1933.

[6] D. Marques, The Fibonacci version of the Brocard-Ramanujan Diophantine equation, Far East J. Math. Sci. 56: 219-224, 2011.

[7] D. W. Masser, Linear relations on algebraic groups, in "New advances in transcendence theory (Durham, 1986)", 248-262, Cambridge Univ. Press, Cambridge, 1988.

[8] E. M. Matveev, An Explicit Lower Bound for a Homogeneous Rational Linear Form in the Logarithms of Algebraic Numbers II, Izv. Math. 64: 1217-1269, 2000.

[9] L. Szalay, Diophantine equations with binary recurrences associated to Brocard-Ramanujan problem, Portugaliae Math., 69: 213-220, 2012.

[10] M. Szikszai, A variant of the Brocard-Ramanujan equation for Lucas sequences, Preprint, 2016.

[11] K. Yu, p-adic logarithmic forms and group varieties II, Acta Arith. 89: 337-378, 1999. 
A. BÉRCZES

InStitute of Mathematics, University of DebreCEN

H-4010 Debrecen, P.O. BoX 12, Hungary

Email address: berczesa@science.unideb.hu

YU. F. BILU

Institut De Mathématiques De BordeauX

Université de Bordeaux and CNRS, TALence, France

Email address: Yuri.Bilu@math.u-bordeaux1.fr

F. LUCA

SCHOOL OF MATHEMATICS

UNIVERSITY OF THE WITWATERSRAND

Private BaG X3, Wits 2050, South Africa AND

DEPARTMENT OF MATHEMATICS

FACULTY OF SCIENCES

UNIVERSITY OF OSTRAVA

30 Dubna 22, 70103 Ostrava 1, CZech Republic

Email address: florian.luca@wits.ac.za 